\title{
A QoS Enhancement Scheme through Joint Control of Clear Channel Assessment Threshold and Contending Window for IEEE 802.11e Broadcasting
}

\author{
Chen Chen, ${ }^{1,2}$ Honghui Zhao, ${ }^{1}$ Hongyu Xiang, ${ }^{1}$ Canding Sun, ${ }^{1}$ Jisheng Sui, ${ }^{3}$ Lijun Zhu, \\ Shengda Wang, ${ }^{3}$ Li Cong, ${ }^{3}$ and Yang Zhou ${ }^{4}$ \\ ${ }^{1}$ State Key Laboratory of Integrated Services Networks, Xidian University, Xian 710071, China \\ ${ }^{2}$ Xidian-Ningbo Information Technology Institute, Ningbo 315200, China \\ ${ }^{3}$ Information and Communication Company, Jilin Electric Power Company Limited, Changchun 130021, China \\ ${ }^{4}$ Ministry of Water Resources Information Center, Beijing 100053, China \\ Correspondence should be addressed to Chen Chen; cc2000@mail.xidian.edu.cn
}

Received 13 June 2016; Revised 29 September 2016; Accepted 25 October 2016; Published 29 January 2017

Academic Editor: Asiya Khan

Copyright (C) 2017 Chen Chen et al. This is an open access article distributed under the Creative Commons Attribution License, which permits unrestricted use, distribution, and reproduction in any medium, provided the original work is properly cited.

\begin{abstract}
In a WLAN, when a great many nodes coexist, the network may readily be congested, thus causing packets dropping and network performance degradation. To solve this issue, a lot of schemes have been proposed. However, most of the previous works attempt to avoid the possible channel congestion by controlling the packets generation rate and/or transmitting power of nodes, while the effects of Clear Channel Assessment (CCA) threshold are not well examined. In our paper, a Joint CCA threshold and contending window control algorithm (JCCA) is proposed to avoid channel congestion or reduce the congestion probability of broadcasting in an IEEE 802.11e network. Both the network conditions and the priorities of messages are taken into account to improve the broadcasting performance in our paper. According to the simulation results, it can be concluded that our scheme can significantly increase the network throughput as well as packets delivery ratio and reduce the packet transmission delay compared to the IEEE 802.1le and Adaptive Carrier Sensing-Based MAC Designs (ACSBM) protocol.
\end{abstract}

\section{Introduction}

Over the past few years, our society has incessantly tended towards the use of wireless communications and social networking technologies. In this regard, the IEEE developed the 802.11 standard [1] in order to define a protocol for offering local area interconnectivity between different wireless devices. The importance of Wireless Local Area Networks (WLANs) has grown considerably due to their simplicity of deployment, low cost, and multimedia content support. To further improve the differentiation of services in IEEE 802.11 networks, the IEEE 802.11e [2] amendment was then developed. As a prioritization method, this amendment introduced a new contention-based channel access method, that is, Enhanced Distributed Channel Access (EDCA), to enable Quality of Service (QoS) guarantee for the original CSMA/CA mechanism [3] used in IEEE 802.11 networks.
However, the broadcasting in IEEE 802.11e networks still adopts the Binary Exponential Backoff (BEB) scheme for collisions resolutions, which actually cannot reflect the degree of channel competitions and is unfair to those nodes that did not transmit successfully [4]. Although the IEEE 802.11e standard specifies a set of recommended values of the contention parameters by which the channel competitions among different prioritized services could be alleviated, its static setting is usually unable to meet various QoS requirements in highly complicated and dynamic networks with different traffic loads [5]. For instance, the high network load generally implies an acute channels competition and a high probability of collisions among traffic flows, resulting in the decrease of system performance. On the other hand, under a light network load, the fixed contention parameters may lead to expanded delay and lower channel utilization during channel accessing. Therefore, to achieve a satisfying 
EDCA performance, an adaptive adjustment protocol is very beneficial to balance the requirements between traffic load awareness and protocol complexity according to the network conditions.

Actually, to increase the performance regarding medium access in IEEE 802.11 networks, many efforts have been made before to improve the BEB based schemes [6]. Some authors also proposed to dynamically adapt CW according to the channel congestion state [7] in order to enhance the network throughput. Based on the previous works, it can be found that there are few works trying to improve the system performance regarding the combination of throughput, channel utilization ratio, packets delivery ratio, and average transmission delay at the same time.

To fill this gap, in our paper, a scheme to improve the IEEE 802.11e WLAN broadcasting performance in terms of the above indexes is proposed through the joint control of the CCA threshold and contending windows. As we know for the original CCA mechanism, when one node is transmitting, all other nodes must wait until it finishes. To check whether the channel is busy or idle, a node has to perform a CCA check. The decision is based on the value of the CCA threshold. If the in-band signal energy crosses this threshold, CCA is held busy until the medium energy is below the threshold [8]. In our work, by adaptively setting the CCA threshold and initial value of the contending windows according to the network condition and priorities of messages, the network performance is significantly enhanced. In summary, the contributions of our work are generalized as follows:

(1) At first, we set different initial values of CCA threshold based on different priorities of messages. After that, we use the channel utilization ratio to determine the CCA threshold for a node when transmitting.

(2) Both network condition and priorities of messages are taken into consideration to improve the broadcasting performance in a WLAN, via the joint control of the CCA threshold and contending window.

The remainder of this paper is organized as follows. In Section 2, we outline some related works regarding the performance improvement in WLANs. In Section 3, our proposed joint control algorithm is given with reasonable assumptions. In Section 4, our proposed algorithm has been theoretically analyzed using a 1D Markov chain model. Section 5 gives the numerical results and performance evaluations of our model. Our paper is concluded in Section 6 followed by acknowledgements and cited references.

\section{Related Work}

A classic article by Bianchi [9] presented the notion that the performance of the traditional DCF (Distributed Coordination Function) strongly depends on the network conditions, mainly the minimum contending window and number of active stations. In addition, the author further confirmed that the parameters of EDCA are very important for collisions resolution, where different parameter settings not only determine the choice of services' priorities, but also influence the overall performance of a network [10]. Specifically, under a complicated network condition, the static parameter configuration of EDCA cannot well optimize the system performance [11]. Additionally, some works also demonstrated that the network has a high collision probability in case of heavy traffic load which would lead to an unsatisfying EDCA performance $[12,13]$.

In [14], the authors proposed a scheme to balance the requirement between throughput and fairness through the estimation to the number of completing nodes and the optimal contending window. This scheme proved that the probability of successful transmission of a broadcasted frame could be improved by up to 50\% [15]. In addition, Calì et al. [16] applied the p-persistent backoff algorithm to the IEEE 802.11 networks and showed that it is possible to tune the backoff window size at run time to obtain a capacity very close to the theoretical limit for stations. Their results show that the capacity of the enhanced protocol is very close to the theoretical upper bound [16] in all the configurations analyzed. However, their scheme needs a station to have an exact knowledge of the network contention level which would be very difficult in practice. As a result, a distributed collision resolution scheme, Asymptotically Optimal Backoff (AOB) [17], is proposed based on the estimation of the network contention level in an IEEE 802.11 wireless LAN. The AOB dynamically adapts the backoff window size according to the estimated network load level and guarantees that a WLAN could asymptotically achieve its optimal channel utilization. In [18], the authors proposed a delay-aware self-adaption scheme for the cumulative improvement of both the throughput and the channel access delay at run time. Although the aforementioned works have their cons and pros in terms of system performance improvement under various traffic loads, there are few works studying the schemes to enhance the network performance with respect to the combination of throughput, channel utilization ratio, packets delivery ratio, and average transmission delay in the meantime.

As for the performance optimization upon CCA adjustment, there are also some previous works for IEEE 802.11 networks. Jamil et al. [19] proposed a CCA threshold adaptation scheme to improve the overall throughput in high density WLANs. Their numerical results showed a global gain of $190 \%$ in the aggregate throughput compared to the upper bound assumed by the present MAC protocol. In [20], a patent is proposed to dynamically adjust the CCA threshold depending on the type of interferences detected. Numerical results show that the self-adaption of the CCA threshold can considerably improve the network performance at the cost of a longer delay for the processor node to access the channel. The patent also demonstrates that there is no fixed threshold suited simultaneously to both heavy and light channel loads. Therefore, it is very necessary to introduce the dynamic CCA mechanism into present IEEE 802.11 protocol stacks.

In summary, it can be concluded that it is still an active topic even today to enhance the broadcasting performance with varying network loads via the self-adaption of the IEEE 802.11e protocol. From our perspective, the adjustment of the CCA threshold should take the following principles into account: 
(1) A higher CCA threshold results in more hidden nodes, smaller radio range, and shorter channel access delay.

(2) A lower CCA threshold will correspondingly result in lower spatial multiplexing ratio, reduced bandwidth utilization, longer channel access delay, and smaller collision probability.

(3) Due to the rapid change of the number of nodes, network topology, and environment, instead of being fixed, the CCA of a network should be adapted to the load conditions for network performance improvement.

(4) The CCA threshold should be decided by taking the priorities of messages into account such that the prioritized messages can access the channel at a higher probability.

In our paper, by taking the aforementioned principles into consideration, a model is proposed via the joint control over the contending window and CCA. We first computed the channel utilization ratio according to (1). After that, we set different initial CCA thresholds and contending windows according to the different types of services. Then, the CCA threshold of a specific service is dynamically adjusted according to the observed channel utilization ratio to decide whether the channel is sensed busy or idle. Finally, we set an appropriate CW based on the channel state for a station.

\section{The Algorithm for Joint Control over Clear Channel Assessment Threshold and Contending Window}

In this section, we will describe our proposed algorithm in detail. To implement our algorithm, the channel utilization ratio is used to classify the network conditions into three categories, that is, low utilization, intermediate utilization, and high utilization cases. In our work, different CCA thresholds and contending windows are set up according to different network conditions and QoS requirements. For readability, the proposed algorithm has been framed as three parts as shown in Figure 1: (1) compute the channel utilization ratio to discriminate the present network condition, (2) initialize the CCA threshold and contending window based on the observed network conditions and messages priorities, and (3) adapt the CCA threshold and contending window to the network conditions by following some specific policies, which will be given later. Each process shown in Figure 1 will be described in detail in Sections 3.1, 3.2, and 3.3, respectively.

3.1. Compute the Channel Utilization Ratio. The channel utilization ratio in our work is defined as

$$
U=\frac{\sum_{n}\left(D_{\mathrm{BUSY}}+D_{\mathrm{AIFS}}+D_{\text {Backoff }}\right)}{D_{\mathrm{CCH}}} \times 100 \%,
$$

where $U$ denotes the channel utilization ratio, $n$ is the number of slots, $D_{\text {BUSY }}$ indicates the channel's busy time, $D_{\text {AIFS }}$ is the value of AIFS, $D_{\text {Backoff }}$ denotes the node backoff

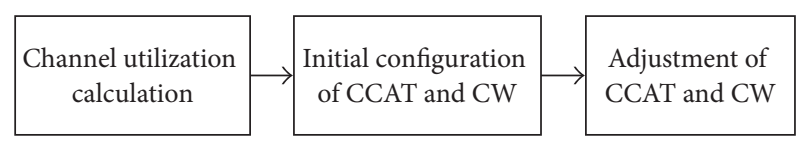

FIGURE 1: Flow chart of our proposed algorithm.

TABLE 1: Configuration of the contending window.

\begin{tabular}{lccc}
\hline $\begin{array}{l}\text { Channel utilization } \\
\text { ratios }\end{array}$ & Network conditions & $\mathrm{CW}_{\min }$ & $\mathrm{CW}_{\max }$ \\
\hline$U<U_{\text {low }}$ & $\begin{array}{c}\text { Low channel } \\
\text { utilization ratio } \\
\text { Intermediate channel } \\
\text { utilization ratio }\end{array}$ & $\mathrm{CW}_{\text {low }}^{\text {low }}(i)$ & $\mathrm{CW}_{\text {min }}^{\text {low }}(i)$ \\
$U>U_{\text {high }}^{\text {mid }}(i)$ & $\mathrm{CW}_{\text {max }}^{\text {mid }}(i)$ \\
\hline $\begin{array}{l}\text { High channel } \\
\text { utilization ratio }\end{array}$ & $\mathrm{CW}_{\text {min }}^{\text {high }}(i)$ & $\mathrm{CW}_{\text {max }}^{\text {high }}(i)$ \\
\hline
\end{tabular}

$i$ is selected from $[0,3]$ denoting four types of services in IEEE 802.11e; $U$ indicates the channel utilization ratio; $\mathrm{CW}_{\mathrm{min}}^{\text {low }}(i)$ is the minimum contending window of the $i$ th type of service when the network is in the low channel utilization case.

TABLE 2: Initial values of the CCA thresholds for different types of services.

\begin{tabular}{lc}
\hline Types of services & Initial values of the CCA thresholds \\
\hline 0 & $\mathrm{CCA}_{0}$ \\
1 & $\mathrm{CCA}_{0}+c$ \\
2 & $\mathrm{CCA}_{0}+2 c$ \\
3 & $\mathrm{CCA}_{0}+3 c$ \\
\hline
\end{tabular}

duration, and $D_{\mathrm{CCH}}$ indicates the executing interval of our control algorithm, respectively. With (1), we then could get the channel utilization ratio while estimating the network congestion level. In our work, the network conditions are classified into three categories according to two predefined utilization ratio thresholds, say $U_{\text {low }}$ and $U_{\text {high }}$.

3.2. Initialize the CCA Threshold and Contending Window. In our work, we define different initial values of the CCA thresholds for messages with different priorities. If the channel utilization ratio is low and the channel resource is underused, a small value of $\mathrm{CW}_{\min }$ should be chosen to increase the probability of the node successfully accessing the channel. On the contrary, if the channel utilization ratio is high, the transmission attempts from nodes are likely to cause the network to be congested. In this case, assigning a larger value of $\mathrm{CW}_{\text {min }}$ may reduce the possibility of collisions. Based on the above analysis, the configuration criterion of the contending windows in our work is listed in Table 1.

To reduce the complexity of our algorithm, we only consider priorities of messages while setting up the CCA threshold and neglect the difference between different network conditions. The setting of the CCA threshold for four different prioritized messages is demonstrated in Table 2.

The CCA thresholds for these four types of messages range from $\mathrm{CCA}_{\text {min }}(i)$ to $\mathrm{CCA}_{\max }(i)$, where $\mathrm{CCA}_{\text {min }}(i)$ and $\mathrm{CCA}_{\max }(i)$ denote the minimum and maximum value of the CCA threshold of the $i$ th type services, respectively. 
3.3. Adjustment of the CCA Threshold and Contending Window. When the node density is low, the channel utilization ratio is usually low and the spectrum resource is underused. In this case, a higher CCA threshold and a smaller $\mathrm{CW}_{\min }$ are preferred for the node to increase the probability of successfully accessing the channel. In our work, if the channel is sensed busy, the contending window is increased by a step value larger than 1 but less than 2 . On the other hand, in the case of the channel being used efficiently, a smaller CCA and a larger $\mathrm{CW}_{\text {min }}$ will be employed to avoid the possible collisions. Accordingly, after the node transmits successfully, instead of directly resetting the contending window to $\mathrm{CW}_{\text {min }}$, we choose a step value to gradually reduce the contending window until it reaches $\mathrm{CW}_{\min }$. The detailed procedure for adjusting the CCA threshold and CW is described as follows.

(1) In the State of Low Channel Utilization Ratio $\left(U<U_{\text {low }}\right)$. Under this state, the number of nodes is generally small and the channel loads are light. To make full use of the network spectrum, a larger CCA and a smaller initial value of $\mathrm{CW}_{\min }$ must be chosen to enable the node to access the channel with a higher probability. Meanwhile, while the channel is sensed busy, the contending window will not be increased in a binary manner, but with by a step value larger than 1 and less than 2 until it reaches the maximum value $\mathrm{CW}_{\max }$, in order to increase the channel utilization ratio. The details of this procedure are given as follows:

(1) At first, the CW of messages with different priorities will be set to their initial values, that is, $\mathrm{CW}^{\text {low }}(i)$. And $\mathrm{CW}_{\min }$ is set to its minimum value, that is, $\mathrm{CW}_{\min }^{\text {low }}(i)$. To make the node access the channel with a higher probability, the CCA threshold is then set to

$$
\mathrm{CCA}_{t}=\mathrm{CCA}_{\max }(i) \text {. }
$$

(2) While the channel is sensed busy, the contending window will not be increased in a binary manner, but by a step value $\alpha$ as

$$
\mathrm{CW}_{\text {new }}^{\text {low }}=\min \left(\mathrm{CW}_{\max }^{\text {low }}(i), \alpha \times \mathrm{CW}\right),
$$

where $\alpha$ is a smoothing factor ranging from 1 to 2 and the $\mathrm{CW}$ is the value of the contending window before sensing the channel. After the $\mathrm{CW}$ is gradually increased to $\mathrm{CW}_{\max }^{\text {low }}$, the $\mathrm{CW}$ will remain unchanged if the channel is still sensed busy. Whenever CW is updated, the node's backoff timer should be recomputed with

$$
T=\mathrm{CW}_{\text {new }} \times \text { Random }() \times \text { Slot Time, }
$$

where $\mathrm{CW}_{\text {new }}$ denotes the value of the contending window when the node backs off again and Random() indicates a function randomly generating a decimal range from 0 to 1 .

(2) In the State of Intermediate Channel Utilization $\left(U_{\text {low }}<\right.$ $\left.U<U_{\text {high }}\right)$. Under this state, the channel is properly utilized, so the original backoff scheme is used for the collision resolution as follows:

(1) At first, the CW of messages with different priorities are set to their initial values, that is, $\mathrm{CW}^{\text {low }}(i)$, and $\mathrm{CW}_{\text {min }}$ is set to its minimum value $\mathrm{CW}_{\text {min }}^{\text {low }}(i)$. The CCA threshold is set to

$$
\begin{aligned}
\mathrm{CCA}_{t}= & \mathrm{CCA}_{\mathrm{min}} \\
& +\frac{U-U_{\text {low }}}{U_{\text {high }}-U_{\text {low }}}\left(\mathrm{CCA}_{\max }-\mathrm{CCA}_{\mathrm{min}}\right) .
\end{aligned}
$$

(2) When the channel is sensed busy, its contending window is doubled in a binary manner as

$$
\mathrm{CW}_{\text {new }}^{\mathrm{mid}}=\min \left(\mathrm{CW}_{\max }^{\mathrm{mid}}(i), 2 \times \mathrm{CW}\right) \text {. }
$$

When CW is gradually increased to $\mathrm{CW}_{\max }^{\text {low }}$, the CW will remain unchanged if the channel is still busy. Whenever CW is updated, the node's backoff timer is recomputed according to (4).

(3) In the State of High Channel Utilization Ratio $\left(U>U_{\text {high }}\right)$. In this case, there are usually numerous nodes contending the channel simultaneously. If the node's contending window is directly restored to $\mathrm{CW}_{\min }$ after a successful transmission, the channel competition will be still acute and the average transmission delays will be expanded with a high risk. Therefore, on this occasion, a higher value of $\mathrm{CW}_{\text {min }}$ should be assigned to each node to alleviate the channel competition. In this way, after a successful transmission, instead of directly resetting the node's contending window to $\mathrm{CW}_{\min }^{\text {high }}(i)$, we halve it step by step. To reduce the collisions among nodes, the CCA threshold must also be set to a smaller value. The corresponding procedure for CCA adjustment under the high channel utilization case is described as follows:

(1) At first, the CWof messages with different priorities are set to their initial values, that is, $\mathrm{CW}^{\text {high }}(i)$. And $\mathrm{CW}_{\text {min }}$ is set to its initial value $\mathrm{CW}_{\min }^{\text {high }}(i)$. Correspondingly, the CCA threshold is set to

$$
\mathrm{CCA}_{t}=\mathrm{CCA}_{\min }(i) \text {. }
$$

(2) When the channel is sensed busy, the contending window is doubled in a binary manner as

$$
\mathrm{CW}_{\text {new }}^{\text {high }}=\min \left(\mathrm{CW}_{\max }^{\text {high }}(i), 2 \times \mathrm{CW}\right) \text {. }
$$

After $\mathrm{CW}$ is gradually increased to $\mathrm{CW}_{\max }^{\text {low }}$, let its value remain unchanged when collisions happen again.

(3) After a successful transmission of the message, instead of directly restoring the node's contending window to $\mathrm{CW}_{\min }^{\text {high }}(i)$, we decrease it linearly via a parameter, that is, 0.5 , in our paper:

$$
\mathrm{CW}_{\text {new }}^{\text {high }}=\max \left(\mathrm{CW}_{\text {min }}^{\text {high }}(i), 0.5 \times \mathrm{CW}\right) .
$$


(1) Input: $D_{\mathrm{BUSY}}, D_{\mathrm{AIFS}}, D_{\text {backoff }}, D_{\mathrm{CCH}}, \mathrm{CCA}_{0}, c$,

(2) Output: $T$ $\mathrm{CW}_{\text {min }}^{\text {low }}, \mathrm{CW}_{\text {max }}^{\text {low }}, \mathrm{CW}_{\text {min }}^{\text {mid }}, \mathrm{CW}_{\text {max }}^{\text {mid }}, \mathrm{CW}_{\text {min }}^{\text {high }}, \mathrm{CW}_{\max }^{\text {high }}$

(3) $U \leftarrow\left(\sum_{n}\left(D_{\text {BUSY }}+D_{\text {AIFS }}+D_{\text {Backoff }}\right) / D_{\mathrm{CCH}}\right) \times 100 \%$

(4) If $i==0$ then $\mathrm{CCA}_{t}^{\text {initial }}=\mathrm{CCA}_{0}$

(5) Else if $i==1$ then $\mathrm{CCA}_{t}^{\text {initial }}=\mathrm{CCA}_{0}+c$

(6) Else if $i==2$ then $\mathrm{CCA}_{t}^{\text {initial }}=\mathrm{CCA}_{0}+2 c$

(7) Else if $i==3$ then $C C A_{t}^{\text {initial }}=\mathrm{CCA}_{0}+3 c$

(8) If $U \leq U_{\text {low }}$ then

(9) $\quad \mathrm{CW}_{\min }=\mathrm{CW}_{\min }^{\mathrm{low}}(i)$;

(10) $\mathrm{CW}_{\max }=\mathrm{CW}_{\max }^{\mathrm{low}}(i)$;

(11) $\operatorname{CCA}_{t} \leftarrow \mathrm{CCA}_{\max }(i)$;

(12) If $\mathrm{CCA} \geq \mathrm{CCA}_{t}$ then

(13) $\quad \mathrm{CW}_{\text {new }}^{\text {low }} \leftarrow \min \left(\mathrm{CW}_{\max }^{\text {low }}(i), \partial \times \mathrm{CW}\right)$;

(14) If transmission is successful, then

(15) $\quad \mathrm{CW}_{\text {new }}^{\text {low }}=\mathrm{CW}_{\text {min }}^{\text {low }}(i)$;

(16) Else if $U_{\text {low }}<U<U_{\text {high }}$ then

(17) $\mathrm{CW}_{\min }=\mathrm{CW}_{\min }^{\mathrm{mid}}(i)$;

(18) $\mathrm{CW}_{\text {max }}=\mathrm{CW}_{\text {max }}^{\operatorname{mid}}(i)$;

(19) $\mathrm{CCA}_{t} \leftarrow \mathrm{CCA}_{\min }+\left(U-U_{\text {low }}\right) /\left(U_{\text {high }}-U_{\text {low }}\right)\left(\mathrm{CCA}_{\max }-\mathrm{CCA}_{\min }\right)$;

(20) If CCA $\geq \mathrm{CCA}_{t}$ then

(21) $\quad \mathrm{CW}_{\text {new }}^{\mathrm{mid}} \leftarrow \min \left(\mathrm{CW}_{\max }^{\mathrm{mid}}(i), 2 \times \mathrm{CW}\right)$;

(22) If transmission is successful, then $\mathrm{CW}_{\text {new }}^{\text {low }}=\mathrm{CW}_{\text {min }}^{\mathrm{mid}}(i)$;

(23) Else if $U \geq U_{\text {high }}$ then

(24) $\mathrm{CW}_{\min }=\mathrm{CW}_{\min }^{\text {high }}(i) ; \mathrm{CW}_{\max }=\mathrm{CW}_{\max }^{\text {high }}(i)$;

(25) $\mathrm{CCA}_{t} \leftarrow \mathrm{CCA}_{\text {min }}(i)$;

(26) If $\mathrm{CCA} \geq \mathrm{CCA}_{t}$ then

(27) $\quad \mathrm{CW}_{\text {new }}^{\text {mid }} \leftarrow \min \left(\mathrm{CW}_{\max }^{\text {mid }}(i), 2 \times \mathrm{CW}\right)$;

(28) If transmission is successful, then

(29) $\quad \mathrm{CW}_{\text {new }}^{\text {high }} \leftarrow \max \left(\mathrm{CW}_{\text {min }}^{\text {high }}(i), 0.5 \times \mathrm{CW}\right)$;

(30) End:

(31) $T \leftarrow \mathrm{CW}_{\text {new }} \times$ Random ()$\times$ SlotTime;

Algorithm 1: Dynamical adjustment of CW based on CCA and priorities of messages. Pseudocode of the proposed JCCA.

After $\mathrm{CW}$ is gradually decreased to $\mathrm{CW}_{\min }^{\text {high }}(i)$, let its value also remain unchanged after a successful transmission. Whenever CW is updated, the node's backoff timer is recomputed with (5); that is, $T=\mathrm{CW} \times$ Random ()$\times$ SlotTime.

The pseudocode of our whole algorithm is shown in Algorithm 1. At first, we calculate the channel utilization ratio according to the given parameters and set the initial value of CCA threshold and CW by types of messages and channel conditions. Then, the CCA threshold and CW were adjusted based on observed channel conditions. Finally, we could derive the backoff timer from the value of CW.

\section{Theoretical Analysis}

In this section, we will give out the theoretical analysis regarding our proposed algorithm. We assume that there are $N$ contending nodes in the discussed network. The sending buffers of all stations are saturated; thus, each station always has packets to be sent. Next, we will analyze our algorithm using the 1D Markov chain model [21] as follows.

Let a discrete-time Markov chain $b(t)$ denote the node's backoff timer at time $t . i$ indicates the $i$ th type services. $W_{0}(i)$ denotes the initial contending window for service $i$ which is equal to $\mathrm{CW}_{\text {min }}^{\text {status }}(i)$. The value of $\mathrm{CW}_{\min }^{\text {status }}(i)$ can be one of the $\mathrm{CW}_{\min }^{\text {low }}(i), \mathrm{CW}_{\min }^{\mathrm{mid}}(i)$, and $\mathrm{CW}_{\min }^{\text {high }}(i)$ statuses depending on the observed channel status. We assume the probability $\tau_{i}(i=1,2,3 \ldots)$, which indicates a station transmitting a packet of the $i$ th type service in a virtual $\operatorname{slot} \tau$, is independent of the backoff procedure. Let $\{k\}$ express the state of each node and $k$ indicate the value of the station's backoff timer in the range $\left(0,1,2,3, \ldots, W_{0}(i)-2, W_{0}(i)-1\right)$. Then, the state transition diagram of our discussed scenario can be given as shown in Figure 2.

As Figure 2 shows, the transition probability from state $k$ to state $k-1$ is 1 . When $k$ is equal to 0 , the station begins to transmit data and choose a random backoff timer to access the channel. The one-step transition probabilities are given as follows:

$$
\begin{aligned}
p\{k \mid k+1\} & =1, \quad k \in\left[0, W_{0}(i)-2\right] \\
P\{k \mid 0\} & =\frac{1}{W_{0}(i)}, \quad k \in\left[0, W_{0}(i)-1\right] .
\end{aligned}
$$




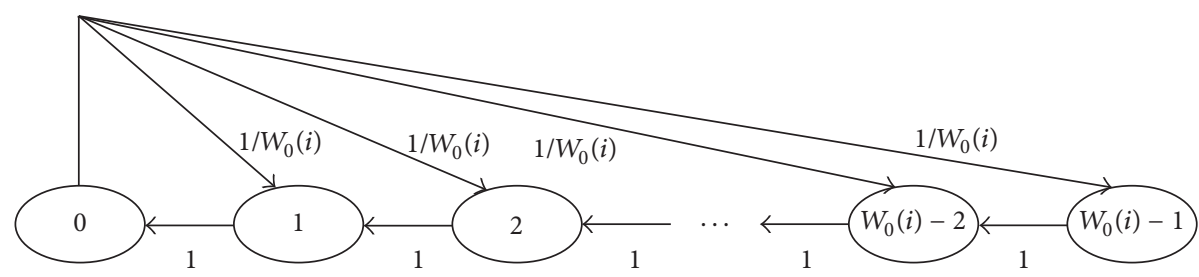

FIGURE 2: State transition diagram.

Let $b_{k}(i)$ be the stationary distribution of the introduced Markov chain which is the node's backoff timer in state $k$. Then, we have

$$
b_{k}(i)=\lim \{b(t)=k\}, \quad k \in\left[0, W_{0}(i)-1\right] .
$$

Therefore, in the steady state, we have

$$
\begin{aligned}
b_{k}(i) & =\frac{W_{0}(i)-k}{W_{0}(i)} b_{0}^{i}, \quad 0 \leq k \leq W_{0}(i)-1 \\
\sum_{k=0}^{W_{0}(i)-1} b_{k}(i) & =1, \quad b_{k}(i) \geq 0 .
\end{aligned}
$$

With (12), we can derive $b_{0}(i)$ as follows:

$$
b_{0}(i)=\frac{2}{W_{0}(i)+1} \text {. }
$$

Since the station will begin to transmit data when the backoff timer is equal to 0 , the probability $\tau_{i}$ of a station transmitting data in any time slot is denoted as

$$
\tau_{i}=b_{0}(i)=\frac{2}{W_{0}(i)+1} .
$$

Therefore, the probability $p_{b}$ that the channel is sensed busy is given by

$$
p_{b}=1-\left(1-\tau_{i}\right)^{n}
$$

Furthermore, the probability $p_{s}$ that a successful transmission occurs within a slot time is derived as

$$
p_{s}=n \tau_{i}\left(1-\tau_{i}\right)^{n-1} \text {. }
$$

Let $p_{c}$ denote the probability that a collision happens. Then, we have

$$
p_{c}=p_{b}-p_{s}
$$

With the aforementioned analysis, once $\tau$ is given, the throughput can be derived as follows:

$$
S=\frac{p_{s} p k}{\left(1-p_{b}\right) \sigma+p_{s} T_{s}+p_{c} T_{c}},
$$

where $p k$ is the MAC payload size (in bits), $\sigma$ is the duration of an empty slot time, $T_{s}$ is the duration during which the channel is still sensed busy due to a successful transmission,
TABLE 3: Simulation parameters of network performance.

\begin{tabular}{lc}
\hline Parameters & Values \\
\hline Size of the beacon message (bytes) & 20 \\
Beacon interval (s) & 5 \\
Propagation model & Two-Ray Ground \\
Array grouping model & $\mathrm{CBR}$ \\
Size of the array group (bytes) & 512 \\
Maximum transmission model $(\mathrm{m})$ & 250 \\
Interface queue & PriQueue \\
Slot time $(\mu \mathrm{s})$ & 9 \\
SIFS time $(\mu \mathrm{s})$ & 16 \\
$U_{\text {low }}$ & $30 \%$ \\
$U_{\text {high }}$ & $70 \%$ \\
Basic data rate & $6 \mathrm{Mbps}$ \\
Data packet generation speed (packets/s) & $1-10$ \\
\hline
\end{tabular}

and $T_{c}$ is the time wasted by a collision, respectively. Note that $T_{s}=T_{c}=\mathrm{DIFS}+\mathrm{DATA}+\mathrm{SIFS}+\mathrm{ACK}$.

Thereupon, the average experienced delay can be estimated as follows. Assume that the total throughput given by (18) is fairly shared by $n$ contending stations; thus, the average packet delay is given by

$$
D=\frac{n \times p k}{S}=\frac{n \times\left(\left(1-p_{b}\right) \sigma+p_{s} T_{s}+p_{c} T_{c}\right)}{p_{s}} .
$$

\section{Performance Evaluation}

Our model is implemented on the NS-2.34 platform [22]. The algorithm is evaluated with the metrics in terms of the average packet transmission delay, packet delivery ratio, and network throughput by varying the vehicular densities. The parameters for network performance evaluation are listed in Table 3. The related thresholds of contending windows referred to in Section 3 are given in Table 4.

In order to accurately evaluate the performance of our proposed model, we have compared our JCCA method with IEEE 802.11e and ACSBM algorithm [23]. The effect of JCCA on packet delivery ratio is shown in Figure 3. It can be noted that, with the increasing of nodes in the network, the packet delivery ratio begins to decline, no matter which method we take. However, the decreasing of the packet delivery ratio of both IEEE 802.11e and ACSBM algorithm is sharper than our JCCA model. Actually, when there are only few nodes contending the network bandwidth, the difference of packet delivery ratio among different methods 
TABLE 4: Setting of the contending window for different message priorities.

\begin{tabular}{|c|c|c|c|c|c|c|}
\hline \multirow[t]{2}{*}{$i$} & \multicolumn{2}{|c|}{$\begin{array}{c}\text { State of low } \\
\text { channel } \\
\text { utilization ratio }\end{array}$} & \multicolumn{2}{|c|}{$\begin{array}{c}\text { State of } \\
\text { intermediate } \\
\text { channel } \\
\text { utilization ratio }\end{array}$} & \multicolumn{2}{|c|}{$\begin{array}{l}\text { State of high } \\
\text { channel } \\
\text { utilization ratio }\end{array}$} \\
\hline & $\mathrm{CW}_{\text {min }}^{\mathrm{low}}$ & $\mathrm{CW}_{\min }^{\text {high }}$ & $\mathrm{CW}_{\min }^{\mathrm{mid}}$ & $\mathrm{CW}_{\mathrm{min}}^{\mathrm{mid}}$ & $\mathrm{CW}_{\min }^{\mathrm{high}}$ & $\mathrm{CW}_{\text {min }}^{\text {high }}$ \\
\hline 0 & 3 & 7 & 7 & 15 & 15 & 31 \\
\hline 1 & 7 & 15 & 15 & 31 & 31 & 63 \\
\hline 2 & 15 & 31 & 31 & 255 & 255 & 1023 \\
\hline 3 & 15 & 31 & 31 & 255 & 255 & 1023 \\
\hline
\end{tabular}

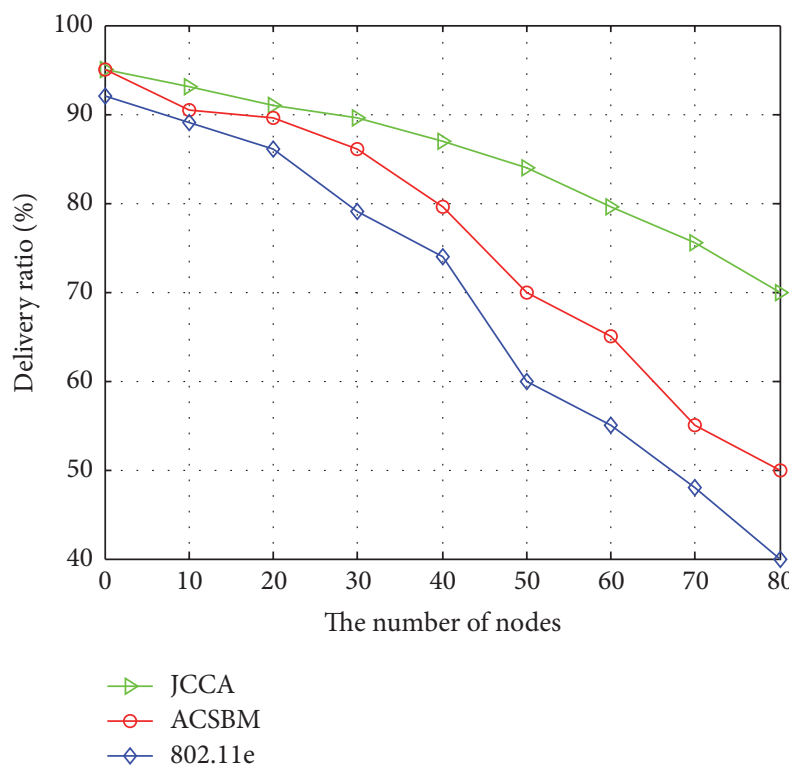

FIGURE 3: Comparison of packet delivery ratio.

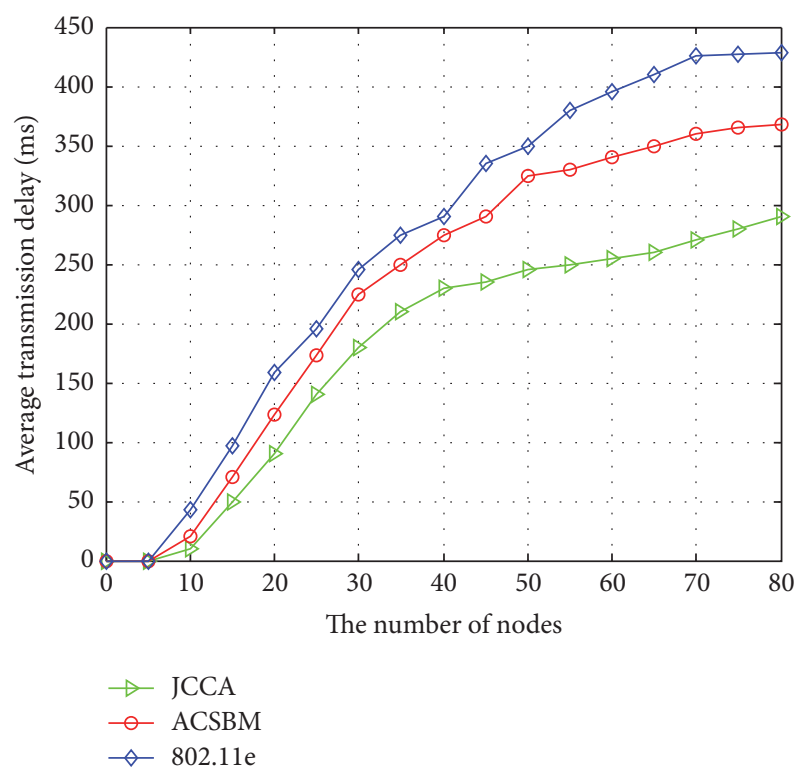

FIGURE 4: Comparison of average transmission delay. is very small. However, when there are a great many nodes in the network, the contending window of IEEE 802.11e and ACSBM will be quickly restored to its initial value after successful transmissions. This makes the access competition among nodes more acute due to heavy network load and then causes the packet delivery ratio to decline. Instead, the CW will decrease gradually to its initial value after successful transmissions using our JCCA protocol, thus reducing the number of contending nodes, as well as increasing the packet delivery ratio.

In Figures 4 and 5, we analyze the impact of our JCCA on average packet transmission delay and network throughput. As shown in Figure 4, at the beginning, the difference of average packet transmission delay among three protocols is smaller. Actually, since only few nodes compete for the channel, the average packet transmission delay will not be expanded too much considering few collisions. When the node density continuously grows, our scheme begins to show the superiority of delay decreasing compared to the other two protocols. Because of lower node density and channel utilization ratio, the contending window of JCCA will not be increased two times but by a parameter larger than 1 and less than 2. In this way, the opportunities for nodes accessing the channel have been increased and the average delay correspondingly decreases. When the node density is higher, unlike IEEE 802.11e and ACSBM, our CCA adaptive and $\mathrm{CW}$ slow decreasing mechanisms not only bring more opportunities for nodes to transmit concurrently but also reduce the number of possible collisions through CW control. In Figure 5, when the number of nodes increases, our JCCA always outperform IEEE 802.11e and ACSBM protocol in throughput. The reason is the same as given for Figure 4, where the CCA adaptive adjustment and load-aware CW control bring more chances for nodes to transmit successfully at the same time, therefore increasing the throughput compared with other two schemes.

To show the impact of our CCA adjustment strategy on the packets reception, we also evaluate the packets reception ratio among three protocols in Figure 6 with radio range or distance varying. As shown in Figure 6, the packet reception ratio reduced with the increasing of radio distance for all three protocols. However, it is worth noting that the reduction of packet reception ratio is slower for JCCA compared to IEEE 802.11e and ACSBM protocols. In fact, the 


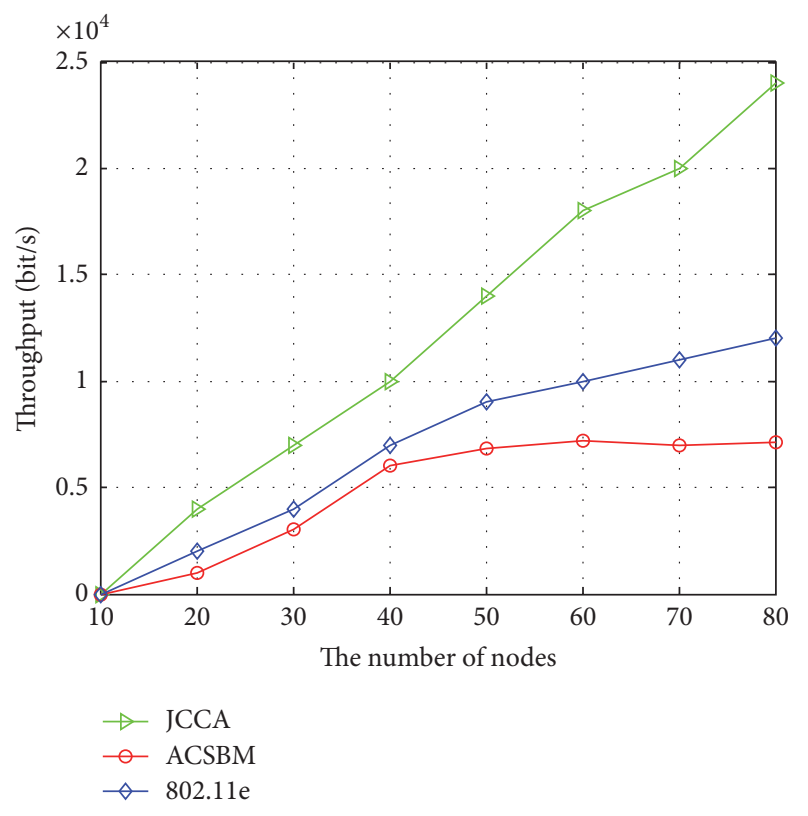

FIGURE 5: Comparison of network throughput.

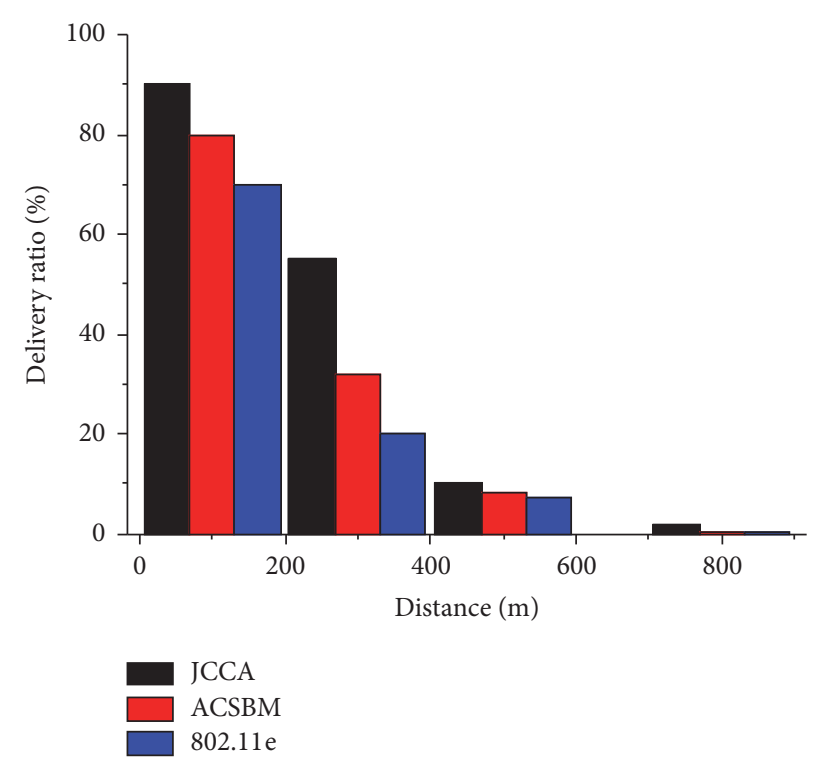

FIGURE 6: Comparison of packets delivery ratio under different radio distances.

adjustment of CCA according to the channel utilization ratio will make our JCCA efficiently use the spectrum resource through intelligent determination of the channel busy/idle state. As a result, although the radio distance setting is directly influencing the packet delivery ratio considering the signal fading and more/few introduced interferences, our JCCA with variable CCA thresholds could outperform the other two protocols at any radio distance.

In Figure 7, the channel utilization ratio of three protocols has been drawn in the box-plot form. A box spans from the first to the third quartile and the median is marked with a line. With the use of congestion or load-aware contention

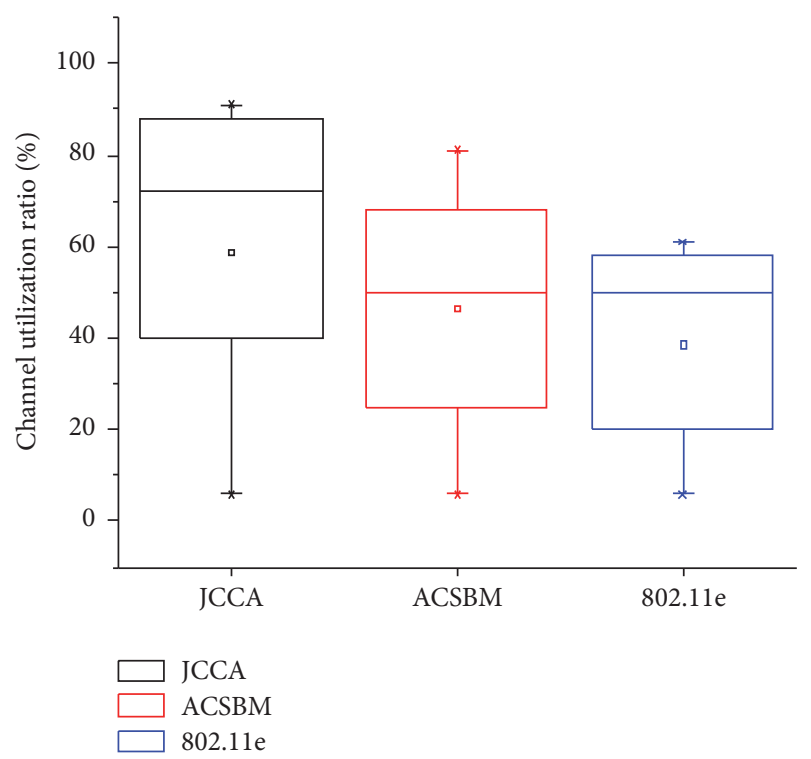

FIGURE 7: Comparison of the channel utilization ratio among three protocols.

strategy, our JCCA shows the highest channel utilization ratio compared with the other two. This means that our JCCA is competent under different load levels and has better scalability considering node densities, transmitting power, channel fading, and so on.

\section{Conclusions}

In the traditional WLANs, the fix setting of the CCA threshold leads to issues such as higher packets collisions and lower channel utilization ratios. In this paper, through the adaptive adjustment of the CCA threshold according to the network conditions and message priorities, the level of spatial reuse is improved by which the performance can be significantly enhanced for an IEEE 802.11e network. In addition, since the load-aware contention resolution is introduced, the channel utilization ratio using our JCCA scheme is improved to avoid network congestion. Numerical results show that our schemes could outperform the IEEE 802.11e and ACSBM in terms of packet delivery ratio, average transmission delay, and throughput.

In our future work, we will study the proactive congestion control schemes via transmitting power and packets generation rate control in high dynamic networks such as VANETs.

\section{Competing Interests}

The authors declare that they have no competing interests regarding the publication of this paper.

\section{Acknowledgments}

This work was supported by the National Natural Science Foundation of China (61201133, 61571338), the National Science and Technology Major Project of the Ministry 
of Science and Technology of China (2015zx03002006003, MJ-2014-S-37), the Natural Science Foundation of Shaanxi Province (2014JM2-6089), the National High-Tech R\&D Program of China (863 Program-2015AA015701), the Ningbo Huimin Projects of Science and Technology (2015C50047), the Research Collaboration Innovation Program of Xian (CXY1522-3), the State Grid Cooperation Project (HX0115014015), and the "111 Project" of China (B08038).

\section{References}

[1] ICSLMS Committee, Wireless LAN Medium Access Control (MAC) and Physical Layer (PHY) Specifications, IEEE Std, 1997.

[2] S. Mangold, S. Choi, P. May, O. Klein, G. Hiertz, and L. Stibor, "IEEE 802.11e wireless LAN for quality of service," in Proceedings of the European Wireless Conference, pp. 32-39, Florence, Italy, February 2002.

[3] A. L. Ruscelli, G. Cecchetti, A. Alifano, and G. Lipari, "Enhancement of QoS support of HCCA schedulers using EDCA function in IEEE 802.11e networks," Ad Hoc Networks, vol. 10, no. 2, pp. 147-161, 2012.

[4] R. Geng, L. Guo, and X. Wang, "A new adaptive MAC protocol with QoS support based on IEEE 802.11 in ad hoc networks," Computers \& Electrical Engineering, vol. 38, no. 3, pp. 582-590, 2012.

[5] S. Keranidis, T. Korakis, I. Koutsopoulos, and L. Tassiulas, "Contention and traffic load-aware association in IEEE 802.11 WLANs: agorithms and implementation," in Proceedings of the International Symposium of on Modeling and Optimization of Mobile, Ad Hoc, and Wireless Networks (WiOpt '11), pp. 334-341, Princeton, NJ, USA, May 2011.

[6] N.-O. Song, B.-J. Kwak, J. Song, and M. Miller, "Enhancement of IEEE 802.11 distributed coordination function with exponential increase exponential decrease backoff algorithm," in Proceedings of the 57th IEEE Semiannual Vehicular Technology Conference (VTC '03-Spring), pp. 2775-2778, Jeju, Republic of Korea, April 2003.

[7] T. Li, T. Tang, and C. Chang, "A new backoff algorithm for IEEE 802.11 distributed coordination function," in Proceedings of the 6th International Conference on Fuzzy Systems and Knowledge Discovery (FSKD '09), pp. 455-459, Tianjin, China, August 2009.

[8] I. Jamil, L. Cariou, and J. F. Helard, "Improving the capacity of future IEEE 802.11 high efficiency WLANs," in Proceedings of the 21st International Conference on Telecommunications (ICT '14), pp. 303-307, Lisbon, Portugal, May 2014.

[9] G. Bianchi, "Performance analysis of the IEEE 802.11 distributed coordination function," IEEE Journal on Selected Areas in Communications, vol. 18, no. 3, pp. 535-547, 2000.

[10] G. Bianchi, I. Tinnirello, and L. Scalia, "Understanding 802.11e contention-based prioritization mechanisms and their coexistence with legacy 802.11 stations," IEEE Network, vol. 19, no. 4, pp. 28-34, 2005.

[11] R. Achary, V. Vaithiyanathan, P. Raj, and S. Nagarajan, "Performance enhancement of IEEE 802.1 le WLAN by dynamic adaptive contention window," in Proceedings of the 16th International Conference on Advanced Communication Technology (ICACT '14), pp. 447-452, IEEE, February 2014.

[12] J. Misic, S. Rashwand, and V. B. Misic, "Analysis of impact of TXOP allocation on IEEE 802.11e EDCA under variable network load," IEEE Transactions on Parallel and Distributed Systems, vol. 23, no. 5, pp. 785-799, 2012.

[13] T. Sanguankotchakorn, A. Gopalasingham, and N. Sugino, "Adaptive channel access mechanism for real time traffic over IEEE 802.11 e Wi-Fi network," in Proceedings of the 4th International Conference on Intelligent Systems, Modelling and Simulation, pp. 486-491, Bangkok, Thailand, January 2013.

[14] M. Luìs, R. Oliveira, L. Bernardo, and R. Dinis, "Maximizing throughput-fairness tradeoff in MAC for ad hoc networks," in Proceedings of the IEEE Wireless Communications and Networking Conference (WCNC '11), pp. 357-362, IEEE, Cancún, Mexico, March 2011.

[15] R. Oliveira, M. Luís, L. Bernardo, and R. Dinis, "Towards reliable broadcast in ad hoc networks," IEEE Communications Letters, vol. 16, no. 3, pp. 314-317, 2012.

[16] F. Calì, M. Conti, and E. Gregori, "Dynamic tuning of the IEEE 802.11 protocol to achieve a theoretical throughput limit," IEEE/ACM Transactions on Networking, vol. 8, no. 6, pp. 785799, 2000.

[17] L. Bononi, M. Conti, and E. Gregori, "Design and performance evaluation of an asymptotically optimal backoff algorithm for IEEE 802.11 wireless LANs," in Proceedings of the The 33rd Annual Hawaii International Conference on System Siences (HICSS-33 '00), Maui, Hawaii, USA, January 2000.

[18] F. Calì, M. Conti, and E. Gregori, "IEEE 802.11 protocol: design and performance evaluation of an adaptive backoff mechanism," IEEE Journal on Selected Areas in Communications, vol. 18, no. 9, pp. 1774-1786, 2000.

[19] I. Jamil, L. Cariou, and J.-F. Helard, "Improving the capacity of future IEEE 802.11 high efficiency WLANs," in Proceedings of the 21st International Conference on Telecommunications (ICT '14), pp. 303-307, Lisbon, Portugal, May 2014.

[20] D. Kloper, D. Chan, and D. Stiff, "Mitigating effects of identified interference with adaptive CCA threshold," Google Patents, 2014.

[21] X. Ma and X. Chen, "Performance analysis of IEEE 802.11 broadcast scheme in Ad hoc wireless LANs," IEEE Transactions on Vehicular Technology, vol. 57, no. 6, pp. 3757-3768, 2008.

[22] T. Issariyakul and E. Hossain, Introduction to Network Simulator NS2, Springer Science \& Business Media, 2011.

[23] M. Derakhshani and T. Le-Ngoc, Adaptive Carrier SensingBased MAC Designs: Throughput Analysis, Springer, 2014. 

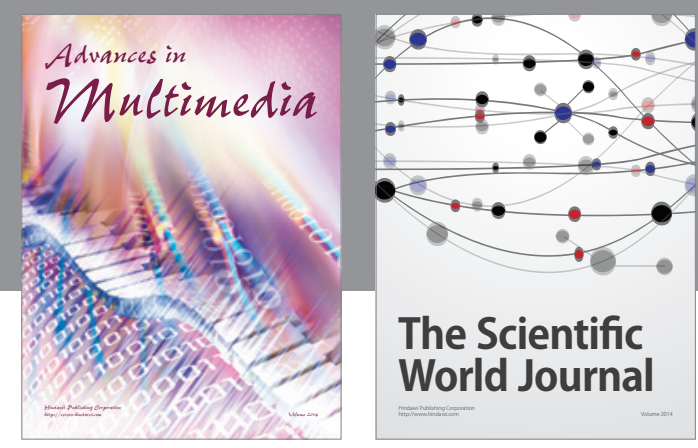

The Scientific World Journal
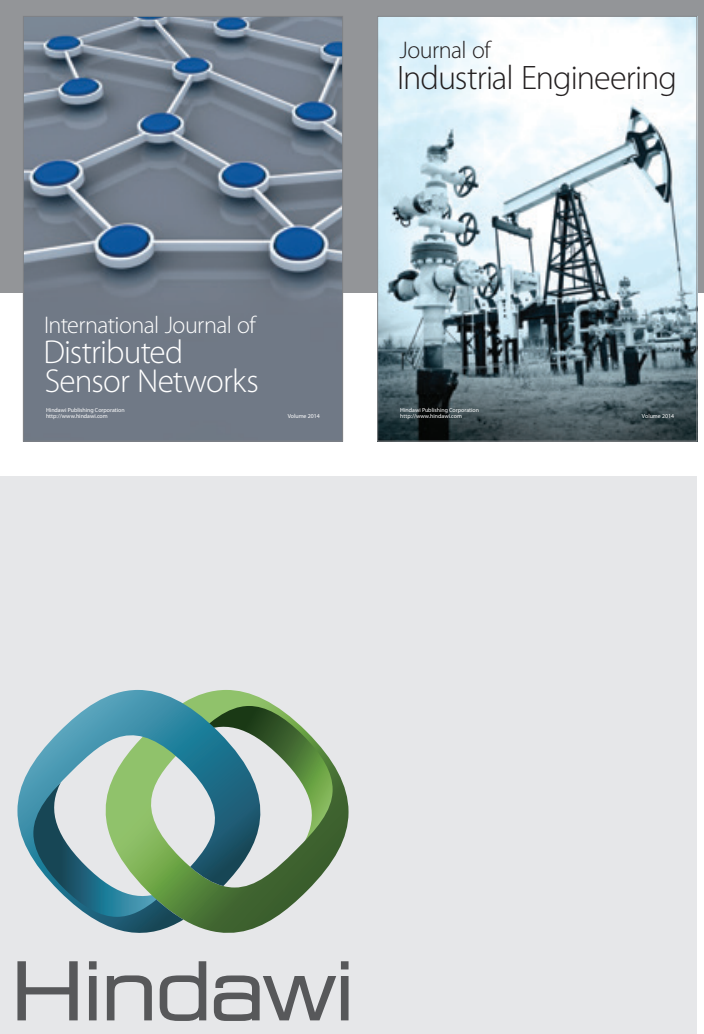

Submit your manuscripts at

https://www.hindawi.com

\section{Computer Networks} and Communications
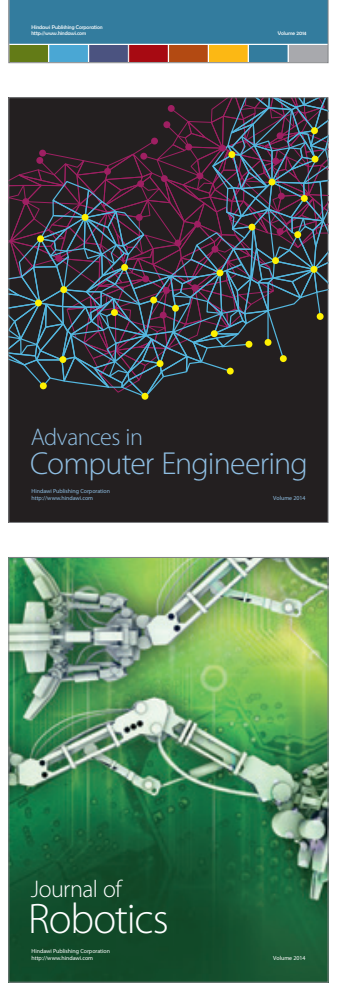
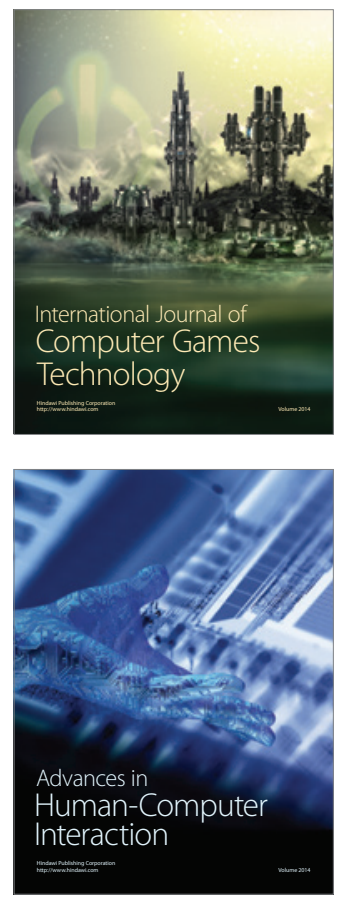
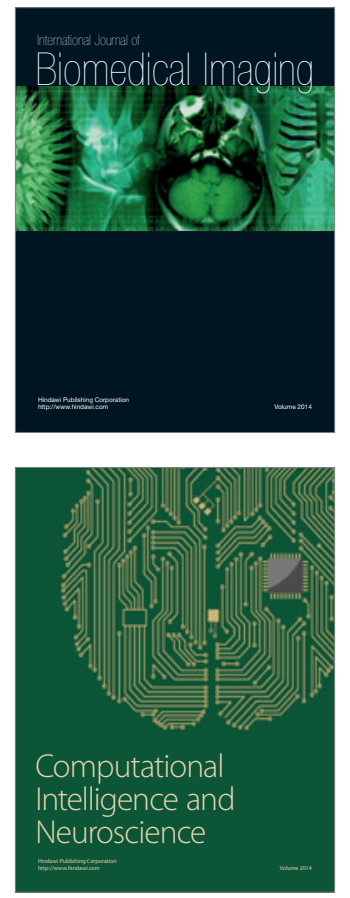
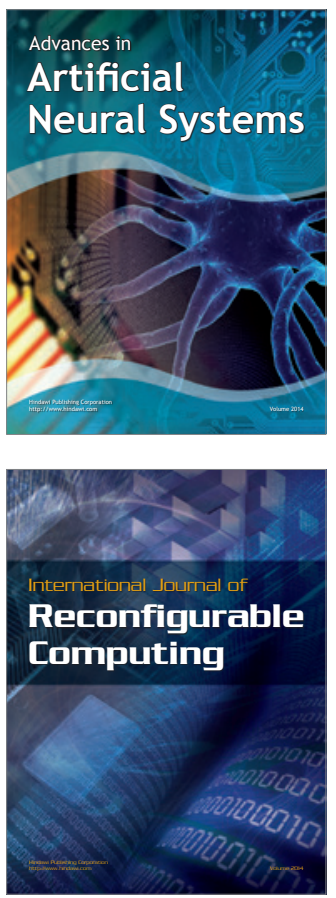
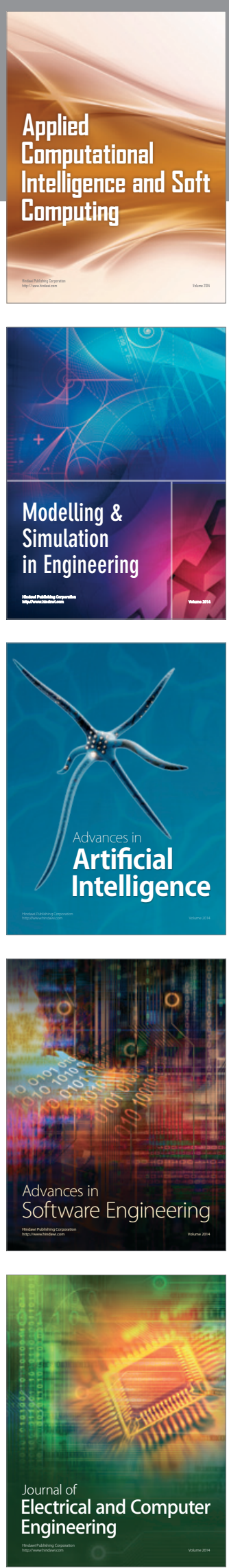